\title{
PROKLA-Redaktion
}

\section{Editorial: Globale Proteste zwischen Organisation und Bewegung}

Die seit 2011 weltweit entflammten Massenproteste überraschten WissenschaftlerInnen wie AktivistInnen gleichermaßen. Trotz der umfassenden sozialen Proteste gegen die Globalisierung in den 2000er Jahren und den Erfolgen dieser Bewegungen insbesondere in Lateinamerika, die Linke an die Regierung zu bringen und Demokratisierungsprozesse auszulösen, hätte vermutlich kaum jemand eine neue Welle sozialer und politischer Bewegungen derartigen AusmaBes erwartet. Drei Jahre später besteht zwar Einigkeit darüber, dass wir es derzeit mit einer historisch besonderen Verdichtung von politischen und sozialen Widerständen und Unruhen zu tun haben. Unklar bleibt jedoch weiterhin, inwieweit es sich tatsächlich um eine globale Protestwelle im Sinne eines transnationalen Bewegungszyklus handelt und wie eng der Zusammenhang zwischen unterschiedlichen pro-demokratischen und kapitalismuskritischen Bewegungen in verschiedenen Ländern und Regionen wirklich ist. Auch die Frage nach den staatlichen Reaktionen und nach reaktionären Tendenzen in diesen Bewegungen ebenso wie nach Gegenbewegungen drängt sich auf. Denn die Proteste gaben gleichzeitig zweideutigen populistischen, rechten oder religiös-fundamentalistischen Kräften Auftrieb. Schließlich gilt es, nach dem Entstehen neuer Verhältnisse zwischen sozialen Bewegungen, linken Organisationen und Parteien sowie der Linken an der Regierung zu fragen.
Ihren Anfang nahmen die Proteste im Dezember 2010 in Tunesien. Nur wenige Wochen später, nach dem Sturz des tunesischen Staatsoberhaupts Ben Ali, breiteten sie sich mit enormer Geschwindigkeit fast im gesamten arabischen Raum aus (vgl. Alnasseri in PROKLA 163,iz3w 324), jedoch mit unterschiedlichen Ergebnissen und Verläufen. In Ägypten führten die Proteste im Februar 2011 zum Zusammenbruch des Mubarak-Regimes; im Jemen wurde durch breite Proteste und bürgerkriegsähnliche Zustände die Regierung abgelöst. In Libyen und Syrien lösten die Proteste Bürgerkriege aus, die zu einer Krise der betroffenen Staaten geführt haben; in anderen arabischen Ländern konnten sie erfolgreich niedergeschlagen und ausgesessen werden.

Die Dynamik der Proteste 2011 erfasste nicht allein die arabischen Länder. Gegen die Krise und Austeritätspolitik kam es zu spektakulären Mobilisierungen in Südeuropa, vor allem in Spanien - eine Protestkonstellation, mit der sich der Beitrag von Nikolai Huke und OlafTietje auseinandersetzt - und Griechenland (Candeias/Völpel 2014: 95ff.), aber auch in Portugal. Im Mai 2011 entwickelte sich aus der Initiative ¡Democracia real YA! die 15M-Bewegung, die überall in Spanien zentrale Plätze gröBerer Städte besetzte und Demonstrationszüge organisierte (Charnock et al. 2011). Nur wenige Tage später kam es zu einem Generalstreik sowie zu Massendemonstrationen in Griechenland und zur Besetzung 
des Syntagma-Platzes in Athen (Kritidis 2012). Fast zeitgleich etablierte sich auch in Israel die J14-Bewegung mit zahlreichen Platzbesetzungen und Zeltstädten, wie Sebastian Schipper in seinem Beitrag zu dieser Ausgabe beschreibt (vgl. Eizenberg in PROKLA 166). Im Herbst 2011 erfassten die Proteste mit der Occupy-Bewegung dann auch die USA und Kanada, nachdem es in Wisconsin bereits im Frühjahr massiven Widerstand gegen die Kürzungspolitik der dortigen Regierung gegeben hatte (Rehmann 2012).

Schon 2010 fanden Proteste von englischen Studierenden gegen die Austeritätspolitik von Cameron statt, im Sommer 2011 kam es zu Unruhen in Tottenham; 2011 protestierten Studierende in Kroatien und Frauen in Italien gegen die Entwürdigung durch Berlusconis Regierung. In Deutschland, wo die von den Krisenbündnissen organisierten Proteste gegen die austeritätspolitische Linie der Regierung Merkel relativ schwach blieben, gab es aber darüber hinaus unerwartet starke Mobilisierungen gegen die nukleare Endlagerstätte in Gorleben und gegen den Bahnhofsneubau in Stuttgart. Auch 2012 setzte sich die Protestdynamik fort. Während es in den Viségrad-Ländern (Ungarn, Tschechien, Slowakei und Polen) und den baltischen Staaten vergleichsweise ruhig blieb, entstanden breite Bewegungen in anderen ost- und südosteuropäischen Ländern wie Bulgarien, Rumänien, Slowenien, Kroatien und Bosnien-Herzegowina, wobei sich letztere auch dezidiert gegen die politische Akzentuierung ethno-nationaler Spaltungslinien richteten, wie Felix Jaitner in seinem Beitrag zu dieser Ausgabe feststellt (für einen Gesamtüberblick vgl. Becker 2013). Auch im subsaharischen Afrika entwickelten sich Protestbewegungen, teilweise - wie in Nigeria - inspiriert von der Occupy-Bewegung (vgl.
PROKLA 170); im kanadischen Quebec formierte sich vehementer Widerstand gegen die Erhöhung von Studiengebühren, in Deutschland, Österreich und anderen europäischen Ländern kam es zu einer starken Bewegung und Selbstorganisierung von refugees, mit denen sich Andrea Plöger in ihrem Beitrag zu dieser Ausgabe auseinandersetzt. 2013, als die Protestwelle vielerorts schon abebbte, entfaltete sich in der Türkei mit der GeziPark-Bewegung (Babacan in PROKLA 173) und in Brasilien mit den Protesten gegen die Fußballweltmeisterschaft (Russau 2014) noch einmal ein neuer Schub in der Protestkonjunktur.

\section{Ein globaler Protestzyklus?}

Die Chronologie dieser Protestdynamik legt einen engen Zusammenhang zwischen den einzelnen Protestereignissen nahe; doch ist allein das zeitliche Zusammentreffen der Protestereignisse noch kein hinreichender Grund, von einem umfassenden Protestzyklus zu sprechen. Allerdings haben die Akteure selbst eine Vielzahl gegenseitiger Bezüge hergestellt: So orientierte sich die J14-Bewegung in Israel bei ihrer Benennung an der 15M-Bewegung in Spanien und übersetzte die Slogans vom Tahrir-Platz ins Hebräische (vgl. Schipper in diesem Heft), die Bewegungen in Griechenland entstanden in unmittelbarer Reaktion auf die Ereignisse in Spanien (Kritidis 2012: 117), viele Protestcamps interagierten durch Live-Schaltungen und transnationale Solidaritätsbekundungen (vgl. Azzelini in diesem Heft). DemonstrantInnen in Wisconsin etwa grüßten die AktivistInnen auf dem Tahrir-Platz, und diese schwenkten Schilder mit transatlantischen Solidaritätsbotschaften, als die US-amerikanische Occupy-Bewegung sich in Oakland mit massiver Repression 
konfrontiert sah. Am 15. Oktober 2011 fand sogar ein "Globaler Aktionstag" statt.

Auch haben sich wichtige übergreifende Themenfelder für die aktuellen globalen Proteste herauskristallisiert: Prozesse der neoliberalen Umstrukturierung, die ihren Höhepunkt in der brutalen Austeritätspolitik finden, mit der die Herrschenden weltweit auf die Finanz- und Wirtschaftskrise von 2007/2008 reagieren, sowie die (damit oftmals eng zusammenhängende) Tendenz zu einer fortschreitenden politischen Entdemokratisierung waren wiederkehrende Motive der Proteste. Besonders deutlich wird die systemische Verschränkung antidemokratischer und neoliberaler Politiken in Griechenland, wo VertreterInnen der Europäischen Zentralbank (EZB), des Internationalen Währungsfonds (IWF) und der EU-Kommission - die berühmtberüchtigte Troika - ihre austeritätspolitische Linie gegen den Willen der Bevölkerung durchsetzten und Druck ausübten, sodass Ministerpräsident Papandreou, der im November 2011 ein Referendum über das Rettungspaket angekündigt hatte, von einer Übergangsregierung abgelöst wurde.

Zugleich waren die Akteure, Gründe, Motive und unmittelbaren Anlässe der Proteste oft unterschiedlich, ebenso wie ihre Protestformen und Entwicklungsmuster - vor allem, wenn man die Bewegungen und Aufstände in unterschiedlichen Weltregionen vergleicht. Entsprechend haben sich parallel zur Entfaltung der Proteste auch verschiedene Interpretationen der Gemeinsamkeiten aktueller Proteste entwickelt. Gängig ist zunächst die Klassifizierung der aktuellen Proteste als Proteste gegen die Krise. So wandten sich gerade in Europa und Nordamerika viele davon gegen die sozialen Auswirkungen der Finanz- und Wirtschaftskrise und ihre austeritätspolitische Bearbeitung. Auch im arabischen Raum und im subsaharischen
Afrika entzündeten sich Proteste oftmals an den im Zuge der Krise steigenden Nahrungsmittel- und Energiepreisen und waren sicherlich motiviert durch die Rückkehr von arbeitslos gewordenen ArbeitsmigrantInnen und ihre nun ausbleibenden Transferzahlungen. Doch der Bezug zur Krise ist nicht immer eindeutig: So richteten sich beispielsweise die Proteste in Ägypten und Israel nicht in erster Linie gegen die unmittelbaren Auswirkungen der Krise, sondern zielten allgemeiner auf die neoliberalen Restrukturierungsprojekte der vergangenen Jahrzehnte (Schipper in diesem Heft, Alnasseri in PROKLA 163). Ähnlich verhielt es sich mit den Protesten in der Türkei, die sich sowohl an der neoliberalen "Modernisierungspolitik“ der Regierungspartei AKP als auch an deren religiöser, wertkonservativer und autoritärer Ausrichtung entzündeten. Zudem erschließt sich aus dem Bezug auf die Krise nicht direkt, warum die Forderung nach Demokratie in vielen Protesten eine derart zentrale Rolle gespielt hat. Vor allem aber bleibt unklar, warum es in Irland und Estland - beides Länder, die mit am schwersten von der Krise im Euro-Raum getroffen wurden - kaum erfolgreiche Mobilisierungen gab, während sich in der Türkei und Brasilien, also in Ländern mit einer auch durch die Krise weitgehend ungebremsten dynamischen wirtschaftlichen Entwicklung, Massenbewegungen ungeahnter Breite entwickelten. Die Krise - soweit sie eng als ökonomischer Einbruch verstanden wird - kann also nur begrenzt als "gemeinsamer Nenner" gelten. Damit bestätigt sich einmal mehr die Einsicht, dass soziale Proteste nicht allein als Reaktion auf Ausbeutung oder Verarmung entstehen.

Ein weiteres verbindendes Moment der Proteste wurde in ihrer sozialstrukturellen Zusammensetzung ausgemacht. 
Gemeinsam sei den Bewegungen, dass sie von einem neuen Klassensegment gut ausgebildeter, junger Menschen mit besonderen socialmedia-Kompetenzen getragen würden, die jedoch keine oder höchstens prekäre Formen der Beschäftigung finden (so u.a. Kraushaar 2012). Das sind wichtige, aber keineswegs hinreichende Ursachen. So verstellt die Annahme eines Generationenkonflikts oftmals eher den politischen Charakter der Proteste, als dass sie ihn erhellt, zumal Jugendliche im arabischen Raum die absolute Mehrheit der Bevölkerung ausmachen (Alnasseri in PROKLA 163). Auch kamen Streiks aus der Arbeiterklasse eine zentrale Bedeutung bei der Initiierung und Entwicklung vieler Proteste zu, wie Torsten Bewernitz in seinem Beitrag zu diesem Heft feststellt. Soziale Medien wie Facebook und Twitter und ihre dezentrale Kommunikationsstruktur waren zwar wichtige Faktoren - gerade unter autoritären Regimen, in denen die traditionellen Medien politisch kontrolliert und zensiert werden. Doch zeigen Forschungen, dass die Nutzung dieser Medien oft erst nach Beginn der Proteste einsetzte, dann aber verstärkend wirkte (vgl. Gerbaudo 2012). Ihre Überbetonung läuft daher Gefahr, die Bedeutung langjährig aufgebauter politischer Strukturen (also Gewerkschaften, linke Parteien und Gruppierungen, zivilgesellschaftliche Netzwerke, Nichtregierungsorganisationen, wissenschaftlich-politische oder künstlerische Zusammenhänge) für die Proteste aus dem Blick zu verlieren.

Auch in anderer Hinsicht lassen sich die Protestbewegungen anhand ihrer Protestformen miteinander vergleichen. Eine übergreifende Gemeinsamkeit der Proteste von Kairo über Madrid, Tel Aviv und Athen bis hin zu New York waren die Besetzung zentraler öffentlicher Plätze, der Aufbau von Camps, die Einsetzung von Asambleas und teilweise die Kommunikation durch das so genannte Human Mic. Wie Henrik Lebuhn in diesem Heft argumentiert, ist die Strategie der Besetzung und Aneignung öffentlicher Plätze jedoch nicht unbedingt das entscheidende Merkmal, das aktuelle Bewegungen von vergangenen unterscheidet - genauso wenig wie die Errichtung von Protestcamps (Leidinger 2012). Darüber hinaus stellt sich die Frage, ob teilweise rechtsgerichtete Bewegungen wie die auf dem Maidan in Kiew dann nicht ebenfalls dem Bewegungszyklus zugeordnet werden müssten. Und schließlich handelt es sich bei den Besetzungen, Camps und Versammlungen zwar um die sichtbarsten Teile der Bewegung, diese sollten jedoch nicht mit den Bewegungen selbst und ihrer oftmals weitverzweigten Infrastruktur verwechselt werden (Candeias/Völpel 2014: 54). Henrik Lebubn schlägt in diesem Heft daher vor, die Gemeinsamkeit eher in der urbanen Dimension vieler Proteste zu suchen. Zwar hätten soziale Bewegungen auch in der Vergangenheit immer wieder den öffentlichen Raum für sich erobert und genutzt. Im aktuellen Kontext beziehen sich die Bewegungen jedoch deswegen so stark auf den städtischen Raum als Ort und Ressource, weil sich in ihm unterschiedliche Dimensionen neoliberaler Politik auf besondere und drastische Weise manifestieren.

Auch im Hinblick auf die Forderungen der Proteste lassen sich Ähnlichkeiten erkennen. So durchzieht die Forderung nach einer „wirklichen" Demokratie viele Bewegungen. Es geht einerseits um die Bekämpfung autoritärer Regimes, gerade im arabischen Raum, andererseits um die Zurückweisung bürgerlicher, „marktkonformer Demokratie“" (Merkel), deren entleerter Charakter insbesondere durch die Politik der Memoranda-of-Understanding-Politik der Troika vorgeführt wird. 
Bemerkenswerterweise gehen die Impulse für eine reale Demokratie in besonders starkem Maße von Protestakteuren aus, die auf eine relativ kurze oder fragile Geschichte der bürgerlichen Demokratie zurückblicken, wie Spanien, Portugal, Griechenland und die Türkei.

Vor dem Hintergrund der breit geteilten Forderung nach 'echter' Demokratie argumentiert Dario Azzelini in diesem Heft, dass die aktuellen Proteste eine wachsende globale Ablehnung liberaler Demokratie und der mit ihr verbundenen Repräsentationslogik sowie die Anwendung alternativer, direktdemokratischer, horizontaler und konsensualer Entscheidungsmechanismen verbindet. Daraus folgt auch eine Ablehnung von Führungsfiguren und politischen Institutionen, die sich nicht nur in einer expliziten Distanz zu sozialistischen oder sozialdemokratischen Parteien, sondern oftmals auch zu Gewerkschaften und anderen klassischen Bewegungsakteuren ausdrückte. Für Azzelini markieren die Massenproteste und Revolten seit Ende 2010 daher einen „Epochenbruch“, vergleichbar mit früheren historischen Zäsuren.

Trotz dieser teilweise komplizierten Verhältnisse von Distanzierung und Kooperation spielten Gewerkschaften in vielen Protesten eine Schlüsselrolle. Während diese in Deutschland im Wesentlichen der Strategie des Krisenkorporatismus folgten, organisierten sie in Südeuropa zahlreiche Generalstreiks. Auch in Slowenien, Tschechien, Polen waren Gewerkschaften für die Initiierung von Protesten zentral. Vor diesem Hintergrund spürt der Beitrag von Torsten Bewernitz in diesem Heft der Frage nach, inwieweit wir es nicht nur mit einem globalen Protestzyklus, sondern auch mit einer globalen Streikwelle seit dem Ausbruch der Krise 2007/2008 zu tun haben und in welchem Verhältnis diese
Arbeitskämpfe zu den bürgerrechtlichen und demokratischen Bewegungen stehen. Dazu gehört die Diskussion darüber, inwieweit die breiten sozialen Proteste gerade in Südeuropa - einen Angriff auf die Organisationsgrundlagen und Rechte der Gewerkschaften mit sich brachten. So zeigen Nikolai Huke und Olaf Tietje in ihrem Beitrag mit Blick auf Spanien, wie die neuen Protestformen die gewerkschaftliche Funktion als Alleinvertretung der ArbeitnehmerInnen infrage stellten. Zu welchen Entwicklungen dieser Widerspruch führt, ob sich daraus Reorientierungs- und Erneuerungsprozesse der Gewerkschaften und ihres Protestrepertoirs ergeben, wie Huke und Tietje es derzeit in Spanien beobachten, oder gar gänzlich neue Innovationsschübe an der Schnittstelle zwischen Organisation und Bewegung, wird zu einer zentralen Frage für die langfristige Bewertung des aktuellen Protestzyklus gehören.

\section{Ein Schritt nach vorne, zwei zurück?}

Aktuell, Ende 2014, sind die großen Protestbewegungen der vergangenen Jahre auf den Straßen und Plätzen kaum noch präsent oder ebben zumindest wieder ab. Im Rückblick wird deutlich, dass die Proteste - von wenigen eindeutigen Fällen abgesehen - zunächst keinen tief greifenden progressiven Politikwechsel erreichen konnten. Im arabischen Raum sind Demokratisierungsprozesse von herrschender Seite weitgehend blockiert worden. In vielen Ländern wurden die Proteste ausgesessen, auch die Austeritätspolitik innerhalb der EU konnte nicht gestoppt werden. Trotz massiver Kritik angesichts der rigorosen Sparprogramme konnten sich die etablierten Parteien an der Macht halten und wurden oft sogar wiedergewählt.

Die Platzbesetzungen erschöpften sich vielfach in der Durchsetzung ihrer eigenen 
Ansprüche: Die horizontalen, direktdemokratischen und konsensorientierten Entscheidungsfindungsprozesse haben sich oft als zeit- und kraftraubend erwiesen. Die "präsentische Demokratie“ (Lorey 2013), die sich der Logik der Repräsentation verweigern möchte, führte zu neuen Ausschlüssen, da nur jene mitbestimmen können, die in der Lage sind, möglichst viel und oft vor Ort zu sein. Gerade subalterne Gruppen, die nicht über die nötige Zeit, die psychische oder physische Konstitution oder die räumliche Nähe verfügen, an den Besetzungen teilzunehmen, konnten nicht einbezogen werden (Candeias/ Völpel 2014: 208). Dies wirft unweigerlich auch die Frage auf, inwiefern beispielsweise der Slogan „We are the 99\%" selbst einen Anspruch auf Repräsentation erheben konnte. Jedenfalls hatten viele Bewegungen Schwierigkeiten, gleichzeitig die Logik der Repräsentation abzulehnen und einen Umgang mit dem Problem zu finden, dass sie selbst immer wieder medial re-präsentiert wurden - zum Beispiel, indem David Grabers Buch Schulden: Die ersten 5000 Jahre als 'Manifest' der Occupy-Bewegung verhandelt wurde.

Wie Azzelini in seinem Beitrag in diesem Heft argumentiert, wäre es jedoch übereilt, diese Entwicklung als Scheitern zu interpretieren. Vielmehr haben sich die Bewegungen 'reterritorialisiert': von den zentralen Plätzen in die Stadteile und die Alltagskämpfe der Menschen. In Spanien entstand die breite Bewegung der Plattform der Hypothekenbetroffenen (Plataforma de Afectados por la Hipoteca, PAH), die das Ziel verfolgt, Delogierungen zu verhindern und Wohnraum zu vergesellschaften (Kasiske 2012). Auch in den USA kam es zu breitem Widerstand gegen Zwangsräumungen, zur Strike-Debt!-Bewegung sowie $\mathrm{zu}$ einer weitverzweigten solidarischen Nachbarschaftshilfe im Zuge von Occupy
Sandy. In Griechenland etablierten sich neue aktivistische Netzwerke, Nachbarschaftszentren und Solidaritätskliniken (vgl. Benos 2014).

Vor diesem Hintergrund, und inspiriert durch den bolivarianischen Prozess in Venezuela, wird insbesondere in Spanien über einen 'konstituierenden Prozess' diskutiert (vgl. arranca 2013): Dieser beschreibt das Projekt, alternative Normen und Institutionen von unten zu entwickeln, mit denen sich die Bevölkerung außerhalb des staatlichen Terrains organisiert und so sukzessive die gesellschaftliche Organisationsmacht des Staates untergräbt. Doch bleibt das Problem, wie verhindert werden kann, dass die Solidaritätsnetzwerke lediglich jenes Vakuum der sozialen Sicherung füllen, das die austeritätspolitischen Angriffe auf die öffentliche Infrastruktur hinterlassen haben; wie also der Gefahr vorgebeugt werden kann, dass zivilgesellschaftliches Engagement zur Stütze neoliberaler Krisenbearbeitung wird - ein Projekt, das (nicht nur) in Großbritannien unter Premierminister Cameron mit dem Schlagwort Big Society von herrschender Seite gezielt vorangetrieben wird (Dowling 2013).

Gegen eine normativistisch-positive Vorstellung von der Zivilgesellschaft stellt sich daher auch die Frage, wie die Bewegungen in die Mechanismen der Aneignung und Verteilung des materiellen Reichtums eingreifen können. Das berührt den Umgang mit staatlicher Macht und Parteipolitik. Auch hier finden Prozesse der Verstetigung der Bewegungen statt, wie die aus der 15M-Bewegung hervorgegangene Partei Podemos in Spanien oder die Initiative für den demokratischen Sozialismus in Slowenien (Korsika/Mesec 2014). In Griechenland stand den sozialen Bewegungen mit SYRIZA von vornherein eine Partei an der Seite, die ihre Strategie auf dem parlamentarischen Terrain in den 
Kontext eines breit angelegten gesellschaftlichen Transformationsprojektes stellt und eine organische Verbindung mit außerparlamentarischen Kräften sucht (Neumann 2014). Als aktuell stärkste Partei verfügt SYRIZA über eine reale Perspektive, in den kommenden Jahren die Regierung in Griechenland zu übernehmen und sich den Auflagen der Troika entgegenzustellen. Entscheidend wird aber sein, ob es SYRIZA in einem solchen Szenario gelingt, angesichts der zahlreichen Übertritte von Mitgliedern der sozialdemokratischen Pasok in die Partei ihren klaren Kurs gegen die Austeritätspolitik beizubehalten und die Bindung zu den Bewegungen nicht abreißen zu lassen.

Wenn oben konstatiert wurde, dass viele Bewegungen derzeit kaum noch durch öffentlichen Protest sichtbar sind, so gilt es gleichzeitig festzustellen, dass sie sich oftmals in einem Prozess von Transformation und Verstetigung befinden und neue, weniger spektakuläre Initiativen verfolgen. Dies trifft freilich nicht nur auf emanzipatorische Strömungen innerhalb der globalen Proteste zu. Auch reaktionäre Kräfte reorganisieren sich derzeit mit erstaunlicher Geschwindigkeit und konnten bereits deutlich an Terrain gewinnen - sowohl im arabischen Raum als auch in Europa. In Ägypten wurde die Bewegung fast vollständig im Machtkampf zwischen den Muslimbrüdern und dem Militär zerrieben, im syrischen Bürgerkrieg in den militärischen Auseinandersetzungen zwischen den Regierungstruppen des Assad-Regimes und islamistischen Kampfeinheiten. In Spanien entwickelte sich eine breite konservative Kampagne gegen die Abtreibung, in Frankreich entstanden Massenproteste gegen die gleichgeschlechtliche Ehe, durch die sich insbesondere der Front National profilierte. Felix Jaitner geht in seinem Beitrag in diesem Heft der Frage nach, warum sich einerseits in Bosnien-Herzegowina eine progressive Bewegung gegen die Austeritätsprogramme entfaltete, während sich andererseits im Nachbarland Kroatien das national-konservative Spektrum als Opposition zum neoliberalen Gesellschaftsmodell etablieren konnte. Er zeichnet nach, wie das national-konservative Lager in Kroatien auf eine direktdemokratische Initiative zurückgriff, um sein zentrales Projekt, die Verhinderung der gleichgeschlechtlichen Ehe, durchzusetzen. Es scheint sich um eine allgemeinere Tendenz zu handeln, die zwei Elemente miteinander verbindet: Zum einen wird ein Kulturkampf entfacht, in dessen Zentrum der Kampf gegen männliche und weibliche Gleichgeschlechtlichkeit und damit verbundene Lebensformen, gegen Sexualaufklärung oder Abtreibung und weitere bevölkerungspolitische Ziele stehen; zum anderen wird die Krise der Repräsentation rechtspopulistisch ausgenutzt, um parlamentarisch oder mit dem verfassungsförmigen Instrumentarium des Referendums, dem der Charakter von plebiszitärer Demokratie verliehen wird, die Demokratie selbst zu schwächen (vgl. Oppenhäuser in PROKLA 171). Das lässt sich mittlerweile in großen Teilen in Europas beobachten.

Neben dem Erstarken reaktionärer Kräfte lassen sich auch eine Brutalisierung beim Einsatz staatlicher Gewaltmittel und eine Aussetzung bzw. schleichende Aushöhlung rechtstaatlicher Elemente beobachten (vgl. Oberndorfer/Caceres 2013 für Spanien). Sebastian Schipper untersucht in seinem Beitrag in diesem Heft, wie in Israel von staatlicher Seite - und jenseits des Einsatzes harter Repression - weitgehend erfolgreich versucht wurde, die Bewegung $\mathrm{zu}$ absorbieren und ihre Forderungen innerhalb eines neoliberalen, marktkonformen Narrativs zu reinterpretieren. 


\section{Kontinuität und Bruch}

Fraglich bleibt, ob sich im Zuge des aktuellen Protestzyklus auch ein neuer kollektiver Akteur herausgebildet hat, wie Michael Hardt und Antonio Negri es mit ihrer Analyse der Multitude nahe gelegt haben. Es lässt sich insofern von einem Protestzyklus sprechen, als die einzelnen Protestereignisse über lokale Konflikte in den jeweiligen Ländern hinauswiesen und sie sich in ihrer Selbstpräsentation wie auch in ihrer medialen Repräsentation zu einem übergreifenden Protestzusammenhang verdichteten. Aufgeworfen wird damit schließlich auch die Frage von Kontinuität und Bruch sowie der Periodisierung von Protestzyklen: In welchem Verhältnis stehen die Proteste zu den Protesten von 1968, den Neuen Sozialen Bewegungen der 1980er sowie zur globalisierungskritischen Bewegung der 2000er Jahre oder den vielfach vorhandenen Protesten und Streikbewegungen, deren historische Konjunkturen und wiederkehrende Auseinandersetzung Immanuel Wallerstein in seinem Beitrag zu diesem Heft nachzeichnet? Wichtig ist in diesem Zusammenhang die Widersprüchlichkeit der Protestdynamiken: Viele der Bewegungen strebten nicht nur eine äuBerst breite gesellschaftliche Orientierung an, wie es die Parole „We are the 99\%" auf den Punkt bringt, sondern gingen oftmals - wie oben bereits ausgeführt - auch auf Distanz zu traditionellen linken Akteuren wie Gewerkschaften und Parteien. Dies ermöglichte die Bildung neuer Akteurskonstellationen, die Entdeckung neuer Themen und die Erfindung neuer Praktiken. So war eine Distanzierung von langanhaltenden politischen Konstellation, aber auch die Ausklammerung zentraler Konfliktfelder wie die Palästinafrage in Israel oder das Verhältnis von türkischer Nation und kurdischer Bewegung bei der Besetzung des
Gezi-Parks eine zentrale Voraussetzung für die Breite der Proteste. Allerdings war der Preis dafür eine gewisse 'Geschichtslosigkeit' und Ausblendung der Kräftekonstellationen. Denn die überkommenen gesellschaftlichen Probleme bleiben ja weiterhin vorhanden; auch die Erfahrungen mit den eigenen Praktiken wurden nicht im Lichte früherer Erfahrungen bewertet. So konnten horizontale, direktdemokratische Entscheidungsverfahren vielfach als Innovation der neuen Bewegungen behandelt werden, obwohl doch deren Bedeutung und Probleme in Auseinandersetzungen wie Avantgarde vs. Massen, Spontaneität vs. Organisierung, Bewegung vs. repräsentative Apparate oder zivilgesellschaftlicher Kampf vs. Kampf innerhalb des Staates bereits seit langem Gegenstand linker Diskussion und Praxis sind. Vor diesem Hintergrund besteht eine zentrale Herausforderung darin, die in den Protesten erprobten neuen Praktiken und Akteurskonstellationen in der Auseinandersetzung mit den Erfahrungen früherer Kämpfe über sich hinauszutreiben, und gleichzeitig ihre Spezifik ernst zu nehmen und zu verstehen. Die vorliegende Ausgabe der PROKLA möchte einen Beitrag hierzu leisten.

\section{Alex Demirović, Henrik Lebuhn, Etienne Schneider (für die Redaktion)}

Mitte Oktober 2014 fand die Mitgliederversammlung der Vereinigung zur Kritik der politischen Ökonomie e.V. statt, der Verein, der die PROKLA herausgibt und die Redaktion und den Redaktionsbeirat wählt. Markus Wissen verstärkt zukünftig die Redaktion und Ingo Stützle löst Michael Heinrich als geschäftsführender Redakteur ab. Sabine Nuss und Stephan Lessenich wechseln von der Redaktion in den Beirat, der zudem von Wolfgang Schöller erweitert wird. 


\section{Literatur}

arranca (2013): Konstitution und Ausdauer. Bewegungen, konstituierende Macht und der Tag danach. Nr. 47.

Becker, Joachim (2013): Krise, Anti-KrisenPolitiken und soziale Proteste in Osteuropa. In: $Z$-Zeitschrift marxistische Erneuerung 25(4): 114-136.

Benos, Alexis (2014): Austerity Kills. Warum die Solidarischen Kliniken auch Orte einer Reorganisierung der Linken sind. In: $L u$ Xemburg 5(1): 58-61.

Candeias, Mario/Völpel, Eva (2014): Plätze sichern! ReOrganisierung der Linken in der Krise. Zur Lernfäbigkeit des Mosaiks in den USA, Spanien und Griechenland. Hamburg.

Charnock, Greig/Purcell, Thomas/RiberaFumaz, Ramon (2001): Indignate!: The 2011 popular protests and the limits to democracy in Spain. In: Capital and Class 36(1): 3-11.

Dowling, Emma (2013): Vom sozialen Wert. Austeritätspolitik, Big Society und die Krise in Großbritannien. In: LuXemburg $4(1): 118-121$.

Informationszentrum 3. Welt (iz3w) (2011): Generation 2011. Revolte in der arabischen Welt. Heft 324.

Gerbaudo, Paolo (2012): Tweets and the Streets: Social Media and Contemporary Activism. London-New York.

Kasiske, Florian (2012): Breiter Widerstand gegen Zwangsräumungen in Spanien. Die Afectados de la Hipoteca wehren sich gegen die Banken. In: ak - analyse \& kritik Nr. 571, 20.4.2012: 23.

Korsika, Anej/Mesec. Luka (2014): Slovenia: From spontaneous protests to renewal of the Socialist Left. In: Kurswechsel 1/2014: 80-88.

Kraushaar, Wolfgang (2012): Aufrubr der Ausgebildeten. Vom Arabischen Frübling zur Occupy-Bewegung. Hamburg.

Kritidis, Gregor (2012): Die Demokratie in Griechenland zwischen Ende und Wiedergeburt. In: Henninger, Max/Birke, Peter (Hg.): Krisen Proteste. Beiträge aus Sozial. Geschichte Online. Hamburg: 101-131.

Leidinger, Christiane (2012) Potenziale politischen Zeltens. Alte und neue Camps als Aktionslaboratorien. In: LuXemburg 3(4): $110-117$.

Lorey, Isabell (2013): Präsentische Demokratie als konstituierender Prozess. In: arranca $\mathrm{Nr}$. 47: 49-54.

Neumann, Mario (2014): Die Welt verändern und die Macht übernehmen. In: ak - analyse \& kritik Nr. 593, 15.4.2014: 25-26.

Oberndorfer, Lukas/Caceres, Imayna (2013): Verlangt das Gesetz der bürgerlichen Sicherheit die Einschränkung der politischen Freiheiten? Spanien und die Neuzusammensetzung von Zwang und Konsens im autoritären Wettbewerbsetatismus. In: Juridikum 25(4): 453-463.

Rehmann, Jan (2012): Occupy Wall Street und die Hegemoniefrage. Eine gramscianische Analyse. In: Das Argument. Nr. 300: 897-909.

Russau, Christian (2014): Die Juni-Proteste. In: Dilger, Gerhard et al. (Hg): Fußball in Brasilien. Widerstand und Utopie. Hamburg: 146-158.

Schmalz, Stefan/Weinmann, Nico (2014): Zwischen Macht und Ohnmacht. Gewerkschaftliche Krisenproteste in Westeuropa. In: Kurswechsel 1/2014: 21-33. 